\title{
Aktuelle randomisierte Kontrollstudien zur Wirkung von Tanz bei Älteren und auf affektive Symptome
}

\author{
Iris Bräuninger
}

I n jüngster Zeit dokumentiert eine Vielzahl von randomisierten Kontrollstudien die Wirksamkeit von Tanz bei unterschiedlichen Symptomen. Dieser Artikel beschäftigt sich mit der Wirkung von Tanz bei älteren Menschen mit nächtlicher Unruhe, Gleichgewichtsproblemen und Sturzrisiko und bei Erwachsenen mit affektiven Symptomen.

\section{Tanz für ältere Menschen mit nächtlicher Unruhe}

Krampe und KollegInnen (2013) untersuchten bei neun älteren Heimbewohnerinnen, ob Tanztherapie dazu beiträgt, das Fortschreiten der funktionellen Einschränkungen zu verlangsamen. Hierzu wurden passive Sensoren über einen kontinuierlichen 2-Monats-Zeitraum verwendet, um nächtliche Ruhe-Muster der Teilnehmerinnen zu messen. Die Heimbewohnerinnen wurden der Tanztherapie-Intervention $(n=5)$ und der Kontrollgruppe ohne Tanzintervention ( $n=4)$ zufällig zugeordnet. Die Tanztherapie-Gruppe nahm dreimal wöchentlich über sechs Wochen hinweg an einer manualisierten Tanztherapie-Intervention zu 45 Minuten teil. Beim Nach-Test zeigten die Tänzerinnen im Vergleich zu den Nicht-Tänzerinnen eine signifikante Verbesserung der Nachtruhe.

\section{Tanz für ältere Menschen mit Gleich- gewichtsproblemen und Sturzrisiko}

Die Studie von Borges und KollegInnen (2014) untersuchte den Einfluss eines Gesellschaftstanz-Programms auf die Balance und die Sturzfrequenz von institutionalisierten älteren Heimbewohnern einer Langzeitinstitution. Nach dem Zufallsprinzip wurden die 59 TeilnehmerInnen der Gesellschaftstanz-Versuchsgruppe $(n=30)$ und der Kontrollgruppe (KG, $n=29)$ zugewiesen. Die
Tanzintervention (Foxtrott, Walzer, Rumba, Swing, Samba und Bolero) fand dreimal pro Woche zu 50 Minuten über einen Zeitraum von 12 Wochen statt. Aus den Patientenakten wurden die Anzahl der Stürze der letzten drei Monate vor der Intervention bestimmt. Beim Pre- und Post-Test-Vergleich wies die Tanzinterventionsgruppe deutlich weniger Stürze beim Post-Test als die Kontrollgruppe auf ( $p<0,0001)$. Die Autorlnnen schlussfolgern, dass ein Gesellschaftstanz-Programm die Balance von älteren, vorwiegend sitzenden Menschen verbessern und die Anzahl der Stürze reduzieren kann.

\section{Tanz für Erwachsene mit affektiven Symptomen}

Pinniger et al. (2013) untersuchten die Wirksamkeit eines zweiwöchigen Intensiv-Programms mit viermal wöchentlich 90 Minuten Tango-Intervention auf selbst berichtete Stress-, Angst- und / oder Depressions-Gefühle ( $\mathrm{N}=41)$. Nach der Randomisierung nahmen 21 Personen an der Tango-Gruppe und 20 an der Kontrollgruppe teil. Im Vor- und Nachtest und im 1-Monats-Katamnese-Test wurden selbstbewertete Symptome von Depression, Angst, Stress, Schlaflosigkeit, Selbstwirksamkeit, Lebenszufriedenheit und Achtsamkeit beurteilt. Die Tango-Gruppenteilnehmerlnnen zeigten beim Posttest eine signifikante Reduzierung der Depression, Angst, Stress und Schlaflosigkeit und eine signifikante Erhöhung der Lebenszufriedenheit und der Selbstwirksamkeit im Vergleich zur Kontrollgruppe. Dieser Effekt hielt auch noch beim 1-Monats-Follow-up-Test in Bezug auf Depression, Angst und Stress an.

Eine weitere randomisierte kontrollierte Studie von Vankova und KollegInnen (2014) überprüfte die Auswirkung der Tanztherapie-Intervention 
„EXDASE“ (Exercise Dance for Seniors) auf depressive Symptome bei älteren, im Langzeitpflegeheim institutionalisierten Menschen $(N=162)$ mit einem Wert der Mini-Mental-State-Examination (MMSE) von 15 oder höher. Der Vergleich der Gruppen zeigte, dass sich die depressive Symptomatik in der Interventionsgruppe signifikant verbesserte $(p=0,005)$, während sie sich in der Kontrollgruppe $(n=83)$ weiter verschlechterte.

\section{Schlussfolgerung}

Die Studien liefern Hinweise darauf, dass sich bei älteren Menschen die depressive Symptomatik, die Sturzgefahr und die nächtliche Unruhe durch Tanz reduzieren lassen. Darüber hinaus kann ein Intensiv-Tanz-Programm die Symptomatik von Depression, Angst und Stress bei Erwachsenen verbessern. Zukünftige Studien können die langfristige Wirksamkeit von tanzbasierten Interventionen auf Affekt und psychische und physische Gesundheit älterer Menschen überprüfen.

\section{Literatur}

Borges, E. G.S., de Souza Vale, R. G., Cader, S. A., Leal, S., Miguel, F., Pernambuco, C.S., Dantas, E. H. (2014): Postural balance and falls in elderly nursing home residents enrolled in a ballroom dancing program. Archives of Gerontology and Geriatrics 59, 312-316, http://dx.doi.org/10.1016/j. archger.2014.03.013

Krampe, J., Miller, S. J., Echebiri, C., Rantz, M. J., Skubic, M. (2013): Nighttime restfulness during daytime Dance Therapy. An exploratory study using bed sensors. Western Journal of Nursing Research 36, 362373, http://dx.doi.org/10.1177/0193945913503716 Pinniger, R., Thorsteinsson, E. B., Brown, R. F., McKinley, P. (2013): Intensive Tango dance program for people with self-referred affective symptoms. Music and Medicine 5, 15-22, http://dx.doi.org/ 10.1177/1943862112470143

Vankova, H., Holmerova, I., Machacova, K., Volicer, L., Veleta, P., Celko, A. M. (2014): The effect of dance on depressive symptoms in nursing home residents. Journal of the American Medical Directors Association 15, 582-587, http://dx.doi.org/10.1016/j.jamda. 2014.04.013

\section{Die Autorin}

\section{Dr. Iris Bräuninger}

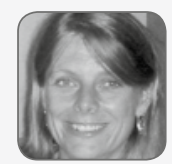

Wissenschaftliche Mitarbeiterin Psychiatrische Universitätsklinik Zürich, Dozentin im Masterstudiengang Tanztherapie, Autonome Universität Barcelona, Supervisorin des deutschen und spanischen Berufsverbandes (BTD, ADMTE), Tanztherapeutin (ADTA), Kestenberg Bewegungsanalytikerin, Psychotherapie (ECP), Private Praxis für Supervision und Therapie.
Dr. Iris Bräuninger Wissenschaftliche Mitarbeiterin Psychiatrische Universitätsklinik Zürich Direktion Pflege, Therapien und Soziale Arbeit Forschung und Entwicklung Lenggstr. 31 | CH-8032 Zürich Tel. 0041 -(0) 443842717 0041-(0)774422676 iris.braeuninger@puk.zh.ch oder dancetherapy@mac.com 